\title{
Public Data Visualization: Analyzing Local Running Statistics on Situated Displays
}

\author{
J. Coenen $^{1}$ (iD) \& A. Vande Moere ${ }^{1}$ (D) \\ ${ }^{1}$ Research[x]Design, KU Leuven, Belgium
}

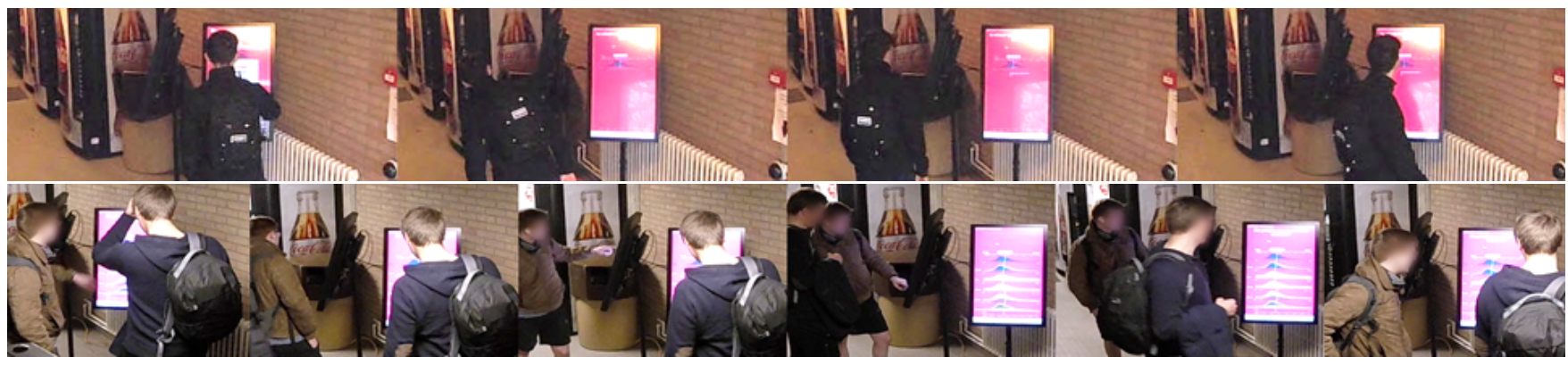

Figure 1: Top: A passer-by, actively engaged with the visualization on one display, briefly inspects another screen. Bottom: Two passers-by, in parallel interaction, moving between displays to support each other in constructing an interpretive frame and exchange insights.

\begin{abstract}
Popular sports tracking applications allow athletes to share and compare their personal performance data with others. Visualizing this data in relevant public settings can be beneficial in provoking novel types of opportunistic and communal sense-making. We investigated this premise by situating an analytical visualization of running performances on two touch-enabled public displays in proximity to a local community running trail. Using a rich mixed-method evaluation protocol during a three-week-long in-the-wild deployment, we captured its social and analytical impact across 235 distinct interaction sessions. Our results show how our public analytical visualization supported passers-by to create novel insights that were rather of casual nature. Several textual features that surrounded the visualization, such as titles that were framed as provocative hypotheses and predefined attention-grabbing data queries, sparked interest and social debate, while a narrative tutorial facilitated more analytical interaction patterns. Our detailed mixed-methods evaluation approach led to a set of actionable takeaways for public visualizations that allow novice audiences to engage with data analytical insights that have local relevance.
\end{abstract}

CCS Concepts

- Human-centered computing $\rightarrow$ Empirical studies in visualization; Visualization design and evaluation methods;

\section{Introduction}

Public displays have become a common technology that allows lay people to engage opportunistically with dynamic information within public space. More than a decade of scientific studies have demonstrated how well-positioned [FH12] public displays showing content that is attuned to the interests of local communities [SFS12] can capitalize on the permeability of public space to promote new types of social interactions and conversations [PTD*20, BGR20]. As a specific public display typology, situated [VMH12] or embedded data [WJD01] representations base their content on the visualization of data of which the referent is located in the imme- diate physical or contextual proximity. Public visualizations have been demonstrated to engage passers-by in data-driven topics with local relevance [CVM13], caused people to self-reflect and socially converse in the context of household energy consumption [VMTH*11, VJTVM13], cycling mobility [CVM17], urban placemaking [TLR*15] and air pollution conditions [CCVM18]. In almost all of these examples, the public visualization lacked meaningful analytical power because the display medium supported little or no interactive features, or because the (often anonymized) data contained too little detail for rich sense-making to occur. To the best of our knowledge, stand-alone public visualizations with analytical 
functionalities have only been studied in well-confined and semipublic contexts such as as museums [HSC11, DMMF20, MMF19] that address a common interest from visitors in data that yet lacks personal purpose.

Encouraged by the rising cultural phenomenon of the quantified self [WK09, Lup16], many athletes are now using smartphones or wearables to capture their personal athletic performance. In recent years, running in particular has grown in popularity [SBB15] because it can be practiced as a leisure activity while enjoying social companionship, rather than to athletically compete [VSBF08, JWT*20]. Studying the analytical visualization of such running performance data on public displays seems apt because it naturally refers to a physical environment around which it can be publicly visualized in a social setting that invites discussion among peers. In addition, crowd-sourced running statistics data now exists on a massive scale, and is relatively openly and knowingly accessible under protection of the European GDPR rules. Moreover, running data is sufficiently situated to be analyzed in relation to a wide range of potential causal factors that can be captured in relatable data, including athlete-specific aspects like gender, training frequency or body weight, or contextual aspects like the time, duration or the weather conditions during a run.

By evaluating a three-week-long deployment of an analytical visualization of running data on two touch-enabled public displays that were located in close proximity to a local community trail, this paper thus investigates: (1) the combination of complementary and established evaluation methods from both public display and visualization research to capture the impact of a public visualization; (2) a public visualization of multi-dimensional data as a facilitator for meaningful analytical interactions; and (3) supporting social data engagement through public visualization. Accordingly, our contributions include: (1) an encompassing mixed-method evaluation approach that captures the analytical and social behaviors surrounding a public visualization; (2) an analytical public visualization shown on two linked, touch-enabled public displays that are able to compare the sports performance statistics of hundreds of runners who frequent the same running trail; and (3) a collection of actionable design considerations that inform future developments in public visualization.

As the visualization field strives to broaden its scope and make data accessible to people that are less "data-savvy" [LCI*20], the potential of using public displays to reach broader cross sections of the population [HTM $\left.{ }^{*} 15\right]$ and those who may not have access to other communication mediums seems obvious. Encouraged by concepts such as the information flaneur [DCW11], a call to explore visualization for communities [TLR $\left.{ }^{*} 15\right]$, and using interfaces beyond traditional desktop environments [RRB $\left.{ }^{*} 11\right]$, this paper helps inform the question whether and how the analytical visualization of data makes sense in opportunistic and public settings.

\section{Related Work}

\subsection{Visualizing Running Data}

The visualization of sports statistics allows people to explore, make sense of, and communicate sports data [PVS*18]. Sports data visualizations can be broadly categorized based on their scope, which ranges from advising individual athletes [KYY*10], over guiding and improving teams [BLC*14], to providing overviews of ranking changes over time to a general audience [PBV09]. Although the potential of personal visualization and visual analytics is being explored [HTA*03], scientific studies on the visualization of running in particular are relatively sparse. Examples include a realtime visualization that supports low-impact running on treadmills [CMHD10] or heatmaps that enable event organizers to design safer trails $\left[\mathrm{OCT}^{*} 08\right]$.

An often forgotten facet is the enjoyable nature of sports, which can be addressed in visualization by reinforcing the emotional connection between an athlete's personal experience and the raw sports tracking data [Woo07]. As such, some running visualizations capture the affective experience of runners through smartphone applications [BHAR16], or provide remote encouragements during long-distance races with ambient and tactile cues [WKBF15]. Moreover, running data has been used as a catalyst for selfreflection, self-improvement and social debate by way of physicalizations designed as 3D objects [STS*12].

\subsection{Visualizing Data on Public Displays}

Based on the degree to which a representation of data is bound to a meaningful referent in physical reality, a visualization can be categorized as 'situated' [VMH12] when it is placed in the context of its data referent, over 'embedded' [WJD01] when its placed directly onto its referent, to 'indexical' [OT15] when the data is revealed by traces of the referent itself. Public visualization can also be considered as a special subcategory of so-called 'pop-up" [FTS17] or 'Internet of Things' [LBV19] interfaces, a type of temporary public display that typically aims to facilitate civic activities such as providing information, collecting opinions, or bringing together locals [PTD*20]. Public visualizations differ in terms of medium, data source and interaction modality. While touch-enabled LCD displays [CVM17] combine both information and interaction, low-resolution e-ink displays [CCVM18] and media architectural facades [BVgSB14] are often accompanied by separate interactive interfaces. In addition, novel carriers have been used to publicly represent data, including passive hand-crafted boards [CVM13], tangible data charts [CM15, PWB20] and biological traces [Off24]. Some public visualizations gather the data that is then conveyed back to the public in order to spark local debate [SFA14, HTM*15, VJTVM13], while others represent external sensor data such as from air pollution [CCVM18] or energy consumption [ $\left.\mathrm{VMTH}^{*} 11\right]$ in order to raise awareness or trigger behavioral changes. Whereas most public visualizations are meant as stand-alone installations, recent endeavours have validated the empowering role of human facilitators to attract passers-by and spark meaningful debates that are based on analytical data [WSP*20] and its broader local implications [PWB20].

Being physically situated in the public environment, any public visualization has to compete for human visual attention. It is known that most passers-by require some physical distance and social opportunity, often in the form of group pressure, to notice and stop for a public display (i.e. 'landing zone" [MWB*12]). Passersby most often do not pay attention to a public display if they do not expect its content to be personally relevant (i.e. 'display blind- 
ness ' $\left.\left[\mathrm{MWE}^{*} 09\right]\right)$. Even then, passers-by often do not realize that a public display is interactive (i.e. 'interaction blindness' $\left[\mathrm{OKK}^{*} 05\right]$ ) or feel socially embarrassed to interact with it when others can perceive them trying [BR03]. Public display research has proposed various models and guidelines to help determine the most ideal audience, content and location of a public display [SFS12], recommendations regarding how these parameters evolve over time and in a community [PTD*20] and the interplay of spatial factors like interaction, social and comfort spaces [FH12].

\subsection{Collaborative and Social Data Analysis}

Visual analytical tasks often benefit from co-located collaboration as they are typically manifold, complex and require large amounts of data. Effective instances of such collaborations around tabletop interfaces can be framed as a spectrum between close and loose types of collaboration [IFP*12]. It has been shown that analytical efficiency can be increased by facilitating discussion around shared large displays [LCBLL16], or by orchestrating multi-device interactions with coordinated multiple views [LHD17]. 'Social data analysis ' uses the ability to share insights online to spark social engagement in casual settings, where distinct user types such as 'killers' and 'explorers' can be identified [HVW09]. As collaborative visualization studies on interactive surfaces are moving to public and casual contexts like museums or music festivals, it becomes clear how strategies like collective play [1KE* 11$]$ or combining guidance with exploratory search [HSC11] positively impact data exploration beyond earlier design considerations for collaborative visual analytics [HA08].

\section{Public Data Visualization Design}

Based on the enthusiastic reactions from casual runners during our pre-design [BCLT14] study at 3 distinct mass-participation running events [CVM20], we realized the potential of an analytical visualization of sports data on a public display. However, accessing the proprietary data from event organizers and deploying and capturing the use of a highly-frequented installation proved technically and organizationally too challenging. To be able to better control and objectively evaluate its impact in a real-world context, we changed the application context from mass-participation events to a more casual and opportunistic indoor space that was located in the near proximity of a running trail, yet that holds large groups of peers with a shared interest in being physically active.

Our visualization was based on Strava data, a popular social platform for tracking running and cycling activity, and its "Segment Leaderboard" data, which is a somewhat competitive yet sufficiently sizable dataset of running performances along trails that were identified by runners. Although this public REST API endpoint was recently removed (API Changes), the dataset was openly accessible and did not require explicit consent from users during the time of this study. For each run, the API provided access to data dimensions like first name and initials, date, time, age and weight categories and gender of the runner. Based on the timestamp, we added attributes that denoted the season and the part of day (i.e. morning, afternoon, evening, night). Results from our predesign study hinted that the final visualization, along with immediate walk-up-and-use usability, should offer: 1) explicit hints to make people aware of the offered interaction possibilities, both in terms of the insights that can be created and the data analytical operations it affords; 2) rich and easily understandable data dimensions that contextualize the dry statistical numbers; 3 ) easily accessible features that promote active hypothesis forming; and 4) more than one display to support concurrent use while diminishing social discomfort and the need to queue.

\subsection{Visual Encoding}

The visualization, illustrated in Fig.2, rendered the arrival time of each run on a particular Strava trail as six vertical ticks, each of which is mapped onto a horizontal scale. Each horizontal scale represented a category, which ordered from top to bottom by assumed relevancy and from broad to specific, included demographic attributes like gender, age and weight group, and contextual factors like the season and the part of the day. Because each run was mapped on an identical, instead of normalized, horizontal time scale, its holistic performance was indicated by a straight vertical line that crossed all the categories and was marked "You" (see Fig.2 B-D). At each intersection with the horizontal scales, a textual annotation lists the absolute ranking of the run in each subcategory. To balance the visual clutter that resulted from overplotting the ticks of hundreds of runs, each category was accompanied by a stacked area chart that illustrated its relative distribution. Although stacked area charts are perceptually limited in terms of comparing individual segments [CM84], they are well liked for their aesthetic appeal and subjectively perceived as more readable than alternatives that provide better performance in terms of correctness and efficiency [TWP*06].

\subsection{Interaction Design}

The interaction design aimed to empower a user to explore how her running performance on the nearby trail ranked in general, in comparison to others in the same subcategories; or in comparison to the average performance of, for instance, a person with another age or weight. The titles across all screens were designed to grab the attention of passers-by with a rotating collection of questions that were deliberately posed as provocative hypotheses, such as "Does weight influence speed?" or "Do men run faster than women?" (see top of Fig.2 A-D). These titles did not affect the visualization itself. The starting screen also featured a text field that allowed users to query a specific run by typing in the first name and initials of a runner. Alternatively, users could select a profile from a predefined collection of profiles, which included the fastest and slowest runner of that week, one of the oldest or youngest, and a random runner. In that case, users were asked to configure their personal running speed (expressed in $\mathrm{km} /$ hour or minutes $/ \mathrm{km}$ ) with a slider.

Consequently, the subsequent screens (see Fig. 2 B-D) always featured a vertical ("You") line that approximated the running performance of a specifically queried runner profile or the manually entered speed, whereas the highlighted subcategories originated either from the queried or predefined runner profile. This screen guided the user through a narrative tutorial, which gradually revealed and explained all the visual features of the first two categories. The reveal of the third category was accompanied by a callto-action, requesting that the user touched any of the segments of 


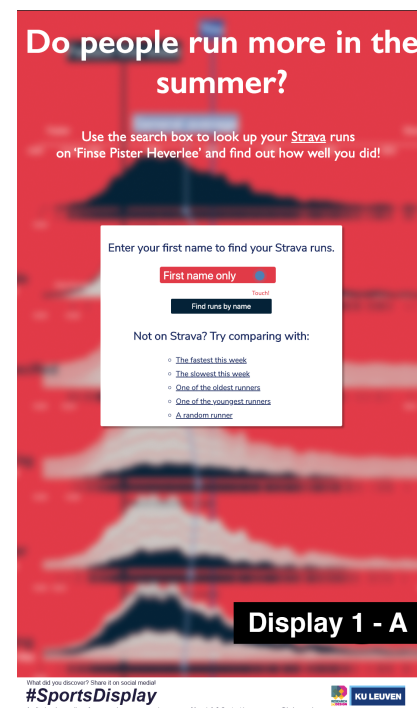

\#SportsDisplay

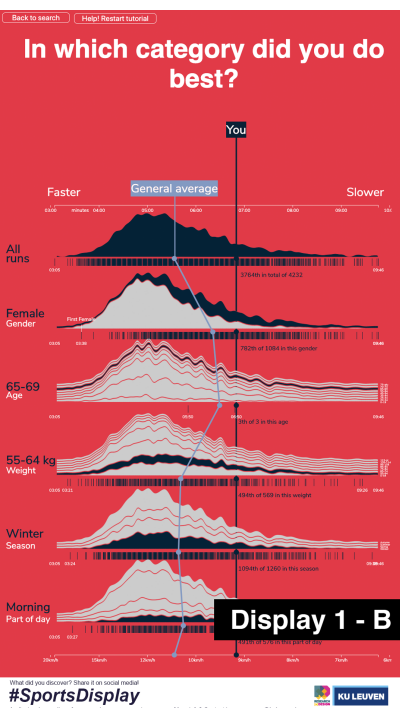

\#SportsDisplay

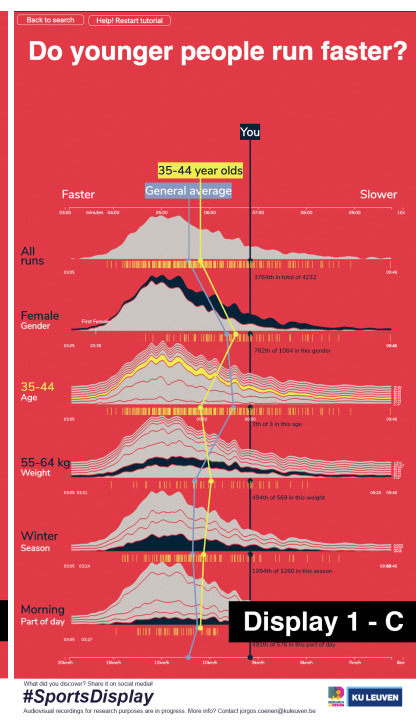

Figure 2: The provocative hypothetical titles at the top aimed to grab attention and act as a creative spark for exploratory analysis. A) Both displays automatically returned to the starting screen after idle time, which allowed passers-by to either enter a specific first name or choose a Strava profile from a predefined list. B) The arrival times of all runs were represented as vertical ticks placed on six horizontal scales, each scale depicted another data dimension in its own category (e.g. gender or age). The relative distribution of arrival times was shown as a stacked area chart with segments corresponding to different subcategories (e.g. female or 65-69 years old). The ticks belonging to the selected run were connected as a straight vertical line ("You"). Area chart segments matching the selected run were colored dark blue, and the corresponding average ticks were connected with a polyline ("General Average"). A textual annotation conveyed the absolute ranking of the chosen run in each selected subcategory. C) Touching a segment of the stacked areas caused all other subcategories to be further filtered on that attribute. $D$ ) On the second display, the vertical line of the run on the first display was denoted with "Other display" to enable cross-display comparison and between-user discussion.

the stacked areas to demonstrate their use as a filter. To support this affordance yet balance visual accuracy, we set a minimum threshold for the thickness of each segment in the stacked area chart algorithm. Once a segment was touched, the remaining three categories were revealed, highlighting the appropriate segments of the stacked areas in dark blue (Fig. 2-B denotes a female, between 65-69 years old, weighing between 55-64 kg, running on a winter morning). After one segment was selected by the user, it should become clear that all segments could be selected as filters. Consequently, by tapping the 35-44 age segment, all the runs from people of that age group were highlighted in yellow on all other active subcategories (as in Fig. 2-C), while a yellow polyline connected the ticks that represented the filtered averages. A second public display showed an identical visualization, with the addition that the run queried on the first display was shown as an additional vertical line labeled "Other Display" (see Fig. 2-D). A video demonstrating the interaction flow can be consulted in the supplemental material available at osf.io.

The visualization was built using web technologies and D3 [BOH11], and deployed on two 32" touch-enabled LCD displays that were positioned at a 90-degree angle to each other to allow concurrent use without ergonomic issues or social conflict. The two public displays are technically described in Fig. 3.

\section{In-The-Wild Study}

The visualization conveyed a total of 3347 runs that were available via the Strava API, 337 of which were added during the study itself. The chosen trail consisted of a $1 \mathrm{~km}$-long illuminated path that is covered with wood-chips to reduce the risk of injury. The displays were deployed during 21 successive days in the side entrance hall of a university building that is positioned directly next to the trail (see Fig. 4). Being occupied by the University Department of Movement Sciences, this entrance hall provides immediate access to publicly accessible sporting facilities, such as showers, exercise spaces, next to teaching and office spaces.

\subsection{Data Collection}

This study took place after receiving approval from our university social and ethics board. All touch-enabled user interactions were digitally logged, including those that occurred with non-interactive elements such as labels. Successive interactions were grouped together to form a single exploration session that originated from one or multiple users. By exaggerating the maximum interval between two successive interactions to 10 minutes, which was the idle time at which the visualization returned to its starting screen, and not separating between the two displays, we intentionally treated consecutive interactions that were triggered by the honeypot effect [BR03] - people naturally encouraging others to join or follow as they are using displays - as single sessions during our analysis. 

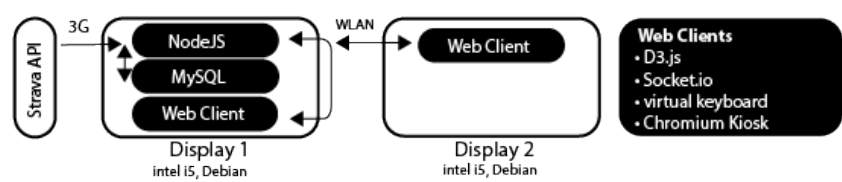

Figure 3: The two public displays each ran on a small computer. Display 1 also functioned as a network server, handling all data updates and their processing.

The environment surrounding the displays was personally observed for approximately 15 hours, and video-recorded for 18.6 hours. Textual messages on the display signaled the possibility of recordings. From this video material, we coded six 45-minute clips, across the different weekdays, times (between 9AM and 10PM), and weeks of the deployment. By comparing with the total of digital $\operatorname{logs}$, we estimate that the analyzed video represents approximately $10 \%$ of interactions. We also made 24-hour-long audio recordings in the immediate vicinity of the installation on 10 weekdays across the study when no video observations were made (total of 164 hours). After recognizing potentially interesting interaction patters on the digital logs, we identified and cross-referenced 63 distinct audio instances. In total, 29 people (10 female, avg. age 23) who interacted with the displays were interviewed in-situ, of which 21 people were spontaneously approached (5-15 minute interviews), and 8 were contacted via Strava after we noticed from the digital $\operatorname{logs}$ that their profiles were queried (20-35 minutes). While quotes from interviews refer to the age and gender of the person (e.g. "F, $24 ")$, quotes from audio recordings do not include such references. The majority of interviewed users $(n=22,75 \%)$ self-identified as a runner.

\subsection{Data Analysis}

The interaction flows were analyzed via the 'Passive-ActiveDiscovery ' model of engagement levels [MLA*12] from public display research by interpreting that 'Passive" engagement corresponds to passers-by observing the displays or physically interacting with them until the starting screen; "Active" with starting the visualization until the tutorial, never filtering or only once; and 'Discovery' with purposefully performing two or more filters or queries. The analytical behavior within 'Discovery' was further examined through analyzing filter patterns and exploration strategies in the digital logs and by grading the insights that were mentioned during the interviews following the insight-reporting methodology from visualization research [SND05]. Independently and in two rounds (reaching $78 \%$ agreement), the first author and one other researcher who was not involved in this study coded 144 insights on depth (i.e. from mere facts to wider contextual interpretations), type (i.e. from based on one data point to relationships across categories) and use of the visual elements (e.g. stacked area charts or annotations). The remaining interview and audio quotes were thematically categorized regarding aspects such as complexity, aesthetics, value and errors in the same manner. A full list of interview questions and themes used for analysis can be consulted in the supplemental material: osf.io.

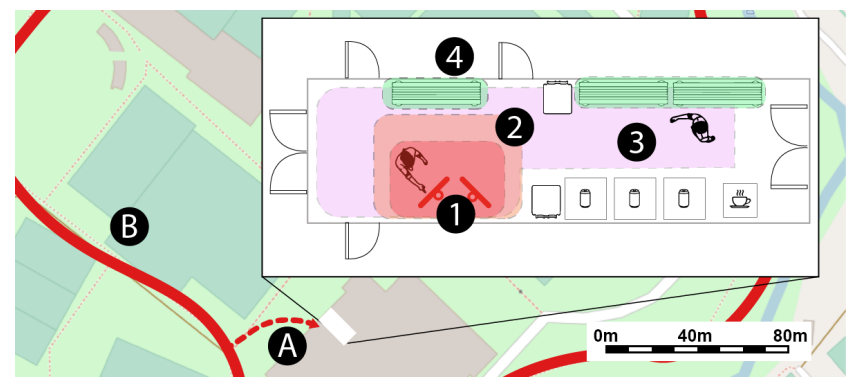

Figure 4: (A) The visualization was situated at the entrance hall of a sports facilities building; (B) located nearby the running trail; which according to [FH12] created (1) interaction, (2) social , (3) activation and (4) comfort spaces.

\section{Results}

\subsection{Passive Engagement}

The digital logs captured 542 visualization starts (daily avg. of 25), from which we derived 235 distinct sessions (daily avg. of 10, see Fig. 5). While an average session lasted approximately 159 seconds, its wide interquartile range of 402 seconds (32s to 434s) reflects the many Passive 'experimental' interactions, such as when users pressed the search button without having entered anything in the text field, and the relatively long exploratory sessions that typically reach 'Discovery' engagement.

Changes in foot traffic were caused by lectures at mornings and early afternoons, causing the narrow space to become too congested to entice passers-by to stop and interact. The "landing zone" [MWB $\left.{ }^{*} 12\right]$ also seemed too short as users often abandoned interacting because other group members continued walking past the displays. While we only observed 4 instances of the honeypot effect leading to additional users, we also noticed 4 instances of what we coin as a detached honeypot effect (see Fig.6), i.e., when the abandoned visualization state on the public display drew attention from passers-by without the presence of any active user.

\subsection{Active Engagement}

$84(34 \%)$ of the sessions engaged with the visualization 'Actively" (see Fig.7) as many users did not continue beyond the tutorial $(n=69)$ or did not touch segments of the stacked areas to apply them as filters $(n=15)$. Our observations show that such "Active" engagements often related to passers-by who only consumed the first part of the tutorial to satisfy their initial curiosity, or became overwhelmed by the overall visual complexity once the tutorial was finished, and then moved on.

Promoting Use. On-site interviews and audio recordings revealed that users engaged with the visualization because they felt encouraged to explore the answers from the hypothetical callsto-action ("Do young people run faster? Em yes, of course!"), or wanted to compare their personal performance ("I'm going to search my name. Yeah you can find yourself!"), that of their acquaintances ("Maybe we should look up [name]?"), or discover noteworthy outliers (You can see who was fastest this week"). Some 


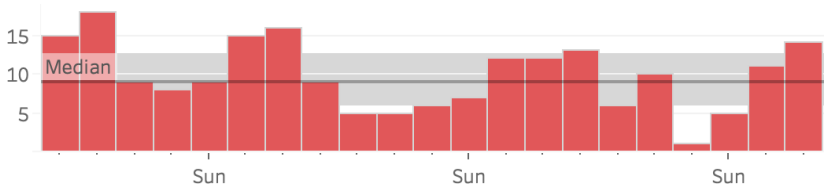

Figure 5: The number of sessions per day shows a slight decline as the study proceeded, with minor peaks at the start of new weeks.

users were attracted by the technological novelty of the installation ("Oh, is it a touch screen? Yeah!"), or felt encouraged because they already used Strava ("It's all just Strava - Still, pretty cool"). Competitive runners were more interested in tracking personal progress ("As a real runner, I'm more focused on my own evolution." M, 21), whereas casual users appreciated comparative features ("Typically if I want to compare within my age, I have to do a lot of 'ctrl-f' inside PDF's" M, 19). Contextual subcategories such as the time of day or season were valued for their novelty ("Season is something extra and fun" M, 21).

Inhibiting Use. Most users reported a mild personal interest in sports data, although they not necessarily wanted to analyze it ("I only run to relax" $\mathrm{M}, 19)$. Some users initially did not want to engage with the visualization because they felt unable to, as they were using a different sports tracking application ("Oh, but I don't use Strava"); or because they considered themselves not to be the target audience as they were not using any sports tracking application ("I just run based on feeling, don't keep track of times" $\mathrm{M}$, 21). Surprisingly, familiarity with the running trail did not create a significant promotor in itself ("Want to see who was the fastest this week? It's all from people doing laps on the trail."), particularly because for most the trail is only part of their run and the visualization alone does not warrant breaking up their activity ("I normally run home, then the app still has to synchronize" M, 25).

Representation. The visual complexity had an inhibiting effect ("Lots of graphs, looks complicated" M, 21), though some appreciated the aesthetics ("Beautiful layout" F, 20) and data density ("A lot of information, I liked that!" M, 24). Users who skipped the tutorial - which occurred regularly (89/235 sessions actively skipped at the start) - felt particularly overwhelmed by the sudden graphical density. Those who followed the tutorial still were startled when the full visualization was revealed ("Suddenly there was a lot!' M, 24). Users suggested the visualization could "give a quick impression once understood" (M, 20) while providing "a good sense of everything through the curves" $(\mathrm{M}, 30)$.

\subsection{Discovery Engagement}

$123(52 \%)$ sessions reached the 'Discovery" stage, as they involved the purposeful use of multiple filters or queries. The majority of these sessions $(n=72)$ reached this stage by following the complete tutorial, and then performing multiple queries $(n=32)$, or actively using filters $(n=40)$. Remarkably, many others $(n=51)$ performed multiple queries, yet only consumed the visualization that was shown in the first step of the tutorial each time (and then immediately returned back to the starting screen), appropriating an analytical strategy we admittedly did not design for.

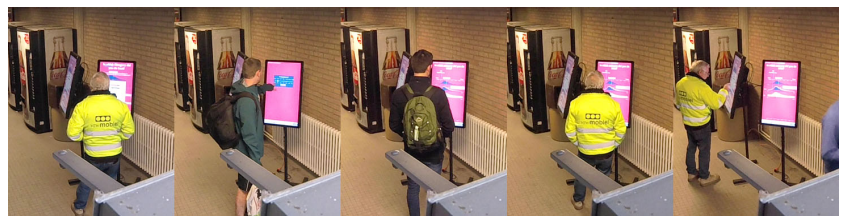

Figure 6: A 'detached honeypot effect' occurred when the abandoned visualization state on the public display persuaded an initially passive observer (left) to actively engage at a later time without the presence of any user nearby (right).

\subsubsection{Data Analytical Strategies}

Exploration. As shown in Fig. 8-A, 8 sessions performed 'deep" filter-driven analyses of only a few runs that were queried. In turn, 19 sessions performed 'shallow' analyses of many different profiles, most of which were instead chosen from the predefined list (Fig. 8-B). One extreme user (Fig. 8-C) looked up a single run and dove deep into different categories. Another user (Fig. 8-D) explored more than 20 predefined profiles on a superficial level, swiftly going back and forth without completing or skipping the tutorial.

Analysis. Using graphical representations similar to Fig. 9, we recognized that the visualization facilitated three common analysis patterns identified in earlier work [GW09] as: 1) a 'Flip' switches filters within the same category (identified in 24 sessions), such as to look for differences within a single category (e.g. influence of age); 2) a "Swap" alternates filters between categories (5 sessions), such as to look for causal correlations between them (e.g. weight impacting men differently than women); and 3) a 'DrillDown" goes through each of the categories one by one (6 sessions), such as to holistically investigate the context surrounding a single run (e.g. filtering based on each relevant subcategory). While we expected individual users to carefully explore their own runs (i.e. 'Drill-Down'), this only transpired in a few sessions in which it typically $(n=4)$ only occurred at the end.

\subsubsection{Explanatory Narrative Tutorial}

In 45 sessions, 2 or more filters were used, of which more sessions $(n=26,58 \%)$ completed the tutorial. Very active filter use (depicted in Fig.10) seems to align with users who also took the time to consume the tutorial. In contrast, only 19 (11\%) out of all 171 sessions wherein the tutorial was skipped led to the use of more than one filter, which had to have been learned prior, through a small textual instruction or by trial and error.

\subsubsection{Contextual Queries over Profile Selection}

Most sessions used the predefined profiles ( $\mathrm{n}=160$ sessions, $77 \%$ ), as only 47 relied solely on entering specific names through the search functionality. Sessions that included multiple profiles were mostly based from the predefined list $(n=97 / 117,83 \%)$. The first listed 'fastest this week' profile was queried most $(n=160$ times in 101 sessions, 39 times as the only query), far outweighing any other profile (between 26 and 57 uses), pointing towards more competitive motivations of analytical use despite the more contextual 


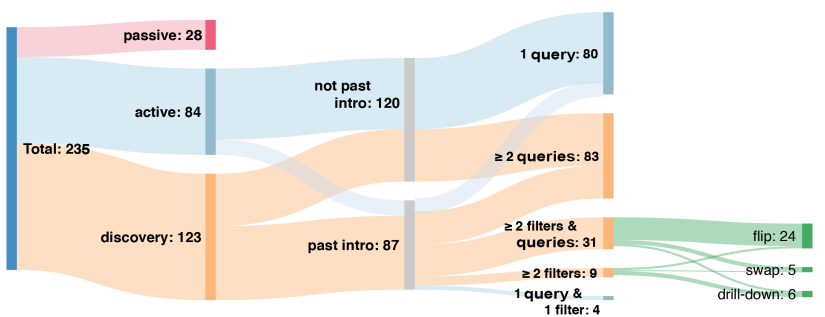

Figure 7: We identify three levels of engagement based on continuation passed the introduction, the number of queries performed and the usage of filters. Most participants who reached 'Discovery' level engagement did so by passing the intro and performing multiple queries (83 sessions).

calls-to-action. 20 identical queries were performed multiple times ( 8 of which spread across 2 to 3 days), suggesting users who returned repeatedly or who learned about performances through word of mouth ("Do you know [name]? He has a really good time, let me show you!"). The most frequently used filters are gender and age $(\mathrm{n}=121$ and 108 respectively). Perhaps surprisingly, seasons are the third most frequently used filters $(n=79)$, even when this category was located at next to last horizontal position.

\subsubsection{Concurrent Display Use}

The digital logs revealed 54 sessions (23\%) during which the two displays were used. Group engagements typical started at one display with a single person interacting while the rest of the group collaborated to construct a common interpretative frame [ $\left.\mathrm{LKH}^{*} 01\right]$ in order to understand the interactive functionalities ("If you use Strava- you can enter your name and see how your runs compare") and encoding ("Those are the really fast ones, and the super slow, and I'm here"). Comparisons that used the 'Other Display" feature were rare, as this label was seldom noticed or understood ("Other display, what does that do?"). Once a common interpretative frame was established, we observed how group members dispersed in three distinct patterns that we coined as 'Hand-Off' (in 19 sessions), 'Parallel' (10) and 'Trial' (6) (exemplified in Fig. 11). 'Trial' interactions typically occurred at a start or end of a session, as members were figuring out how the two displays were linked (see Fig. 1-top). The 'Hand-Off' pattern is indicative of how a single group member moved to the other display to interact for themselves and how the detached honeypot effect provided a frictionless starting point for new users.

\subsubsection{Social Interactions}

The audio recordings revealed that nearly half $(n=31 / 63,49 \%)$ of the captured instances involved discussions between an active user and an acquaintance. Most conversations that occurred in the immediate vicinity of the displays were sparked by three themes: the titles, social context and data source. The hypothetical titles sparked discussions that were overheard from 'Passive" passers-by, as they theorized about potential answers ("Do people run slower in winter? [laugh] Would that make sense?" - "I think it's colder so you can't get hot. Do you run faster than?"). Yet their influence

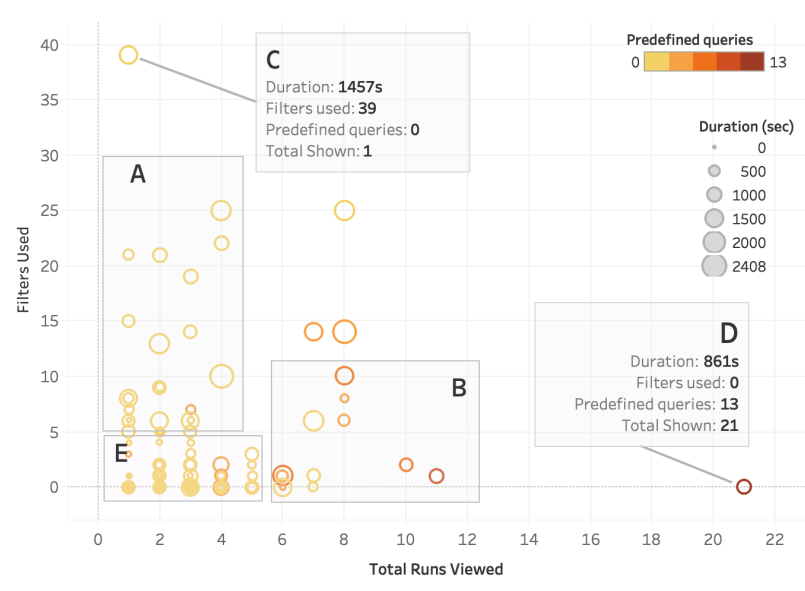

Figure 8: We identified sessions with five distinct analytical interaction strategies based on the balance between filter use and queries. Some sessions $(A, n=24)$ involved deeper explorations of only a few queries, while others $(B, n=19)$ exemplify more shallow analysis of multiple runs. In addition, two extremes can be identified that either extensively explored the context of one run $(C)$, or only performed a cursory interpretation of many runs $(D)$. Finally, the majority of the sessions $(E, n=163)$ observed less than 6 runs and used less than 5 filters. 28 (not depicted) interacted only with the starting screen.

rarely extend into actual analysis $(n=2 / 31)$. Users were motivated to communicate insights that were socially relevant to them. They looked up others they knew, and shared their recent performances ("This is from Saturday's training, see?") or jokingly looking up each other's runs ("Let's see where you land! Oh, out of bounds!"). The data source itself was often discussed in terms of different types of bias, uncertainty [JS09] and implicit error [MGM19]. Passers-by seemed familiar with Strava data and associated it with more competitive athletes ("Strava users are typically quite competitive" F,19), doubting its broader relevance (I'm not sure if it's the best comparison for me as non-runner" F, 24). Users doubted the accuracy of the data due to personal experiences ("I have never updated my weight" M, 20), stories of errors ("If you run with the app on your phone, the GPS can be completely wrong due to the buildings and trees" M, 20) and expectations of cheating ("That has to be someone on a bike!"). Some conversations dealt with the visualization feature to input one's own running speed, as users assumed that at least some of the visualized data was sourced in this way ("Everyone lies", or "I'm going to mess up the average").

\subsubsection{Insight Reporting}

Interviewees were able to remember and describe between 1 and 12 insights (avg. 4.43, SD 2.73), most of which were generated by comparing the vertical line ("You") to the polyline connecting averages in the highlighted subcategories, or the relative thickness of the segments of the area charts (see Table 1). Most users compared data only within categories and using one visual encoding (e.g. "In summer people are faster, that's also my experience" $\mathrm{M}$, 18 , referring to the shifting position of the vertical polyline within 
J. Coenen \& A. Vande Moere / Public Data Visualization: Analyzing Local Running Statistics on Situated Displays

\begin{tabular}{|c|c|c|c|c|c|c|c|c|c|c|c|c|c|}
\hline & \multicolumn{8}{|c|}{ Insight Depth } & \multicolumn{5}{|c|}{ Visual Elements } \\
\hline Average (SD) & $\begin{array}{l}\text { Facts } \\
1.71(1.1)\end{array}$ & $\begin{array}{l}\text { Interpretation } \\
2(1.06)\end{array}$ & $\begin{array}{l}\text { Pers. Refl. } \\
2.06(1.2)\end{array}$ & $\begin{array}{l}\text { Prior Knowl. } \\
1.55(0.99)\end{array}$ & $\begin{array}{l}\text { Contradict / Confirm } \\
1.57(0.72)\end{array}$ & $\begin{array}{l}\text { Physical Context } \\
1.4(0.49)\end{array}$ & $\begin{array}{l}\text { Social Context } \\
1.78(0.79)\end{array}$ & $\begin{array}{l}\text { Data Context } \\
1(0)\end{array}$ & $\begin{array}{l}\text { Annotation } \\
1.1(0.3)\end{array}$ & $\begin{array}{l}\text { Area charts } \\
2(0.92)\end{array}$ & $\begin{array}{l}\text { Polylines } \\
2.29(1.88)\end{array}$ & $\begin{array}{l}\text { Overview } \\
1.33(0.6)\end{array}$ & $\begin{array}{l}\text { Ext. Knowl. } \\
1.66(1)\end{array}$ \\
\hline Total Reported & 24 & 32 & 35 & 17 & 36 & 7 & 16 & 3 & 11 & 38 & 49 & 21 & 25 \\
\hline$\geq 1$ & 14 & 16 & 17 & 11 & 23 & 5 & 9 & 3 & 10 & 19 & 21 & 15 & 15 \\
\hline
\end{tabular}

Table 1: All insights reported during 29 on-site interviews were coded based on depth and the use of the visual elements provided by the visualization. This table documents the average number of each insight type per interview, the total reported insights across all interviews, and the number of interviews with 1 or more matching insight types.

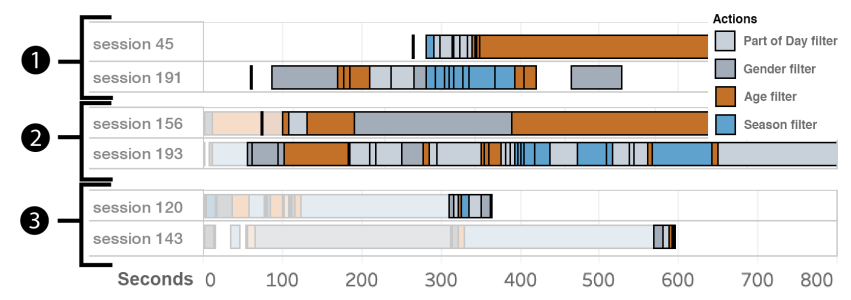

Figure 9: A selection of indicative interaction logs demonstrates the common analysis patterns 'Flip' (1), 'Swap' (2) and 'Drilldown' (3) facilitated by patterns in filtering actions (e.g. gender, age, weight). The width of the actions correspond to the time spent in this state.

one category) whereas few used multiple categories in a single insight (e.g. "In the morning people tend to be slower, as well as the winter, maybe because of cold muscles? M, 20) or multiple encodings (e.g. using the vertical line and segment thickness: "In summer people run less, and in winter they are less fast" M, 21). Over half of the interviewees reported insights that included personal reflections, such as by comparing one's own performance to a subcategory ("Interesting, among women I score a lot better" F, 20) or a predefined profile ("We are slower than the oldest man" F, 19). People reflected on the social context of insights ("There are a lot of active people around, that might skew the impression" M, 21), on the physical (e.g. "Slower in Autumn makes sense, it's swampy now" M, 27) or data context ("Not so great, but Strava users are typically competitive” $\mathrm{F}, 19$ ).

Most users (23 interviews) reported insights in the form of contradictions or confirmations of their expectations. It is also the type of insights that is most reported overall $(n=36)$, suggesting that users had some preceding insight into the topic and context that were verified or debunked by the visualization ("I'm doing better than expected actually" F, 24 or "Expected morning runs to be better, it changed but not much" M, 26). This is further supported by the insights that use the visualization in combination with prior personal knowledge to bolster discoveries ("Many between 4:40 and $6: 20$, those are not trained but hobby runners at chatting tempo" M, 20).

\subsubsection{Usability Issues}

Interviewees who had difficulty interpreting the visualization typically struggled with the (left vs. right) direction of the time axis $(3 / 29)$, the focus on a single lap time instead of an average of multiple runs (2/29), or the meaning behind the selected area segments $(3 / 29)$. These issues occurred particularly when users interpreted a predefined profile of which the relevant subcategories were not personally known to them (e.g. the age of the fastest person that week).

We logged users trying to "slide" the vertical line ("You") although it was not interactive (in 6/235 sessions) and touch the textual labels instead of the stacked areas (26/235 sessions). Although we set a minimum thickness for each segment, those with few data values were hard to select. This issue was most obvious for the age filters, where the smallest subcategories (e.g. age group 70-74 and above 75 ) received very little attention ( $\mathrm{n}=10,9 \%$ of age filters). Although relatively narrow, the segment with the highest weight subcategory (above $95 \mathrm{~kg}$ ) received more than $18 \%$ of attention for this category, which might reflect the persuasive power of some titles (i.e. "Do heavy people run slower?").

\section{Discussion}

Our public displays were able to attract a similar proportion of passers-by as other touch-enabled public displays (i.e. 10 average daily sessions, comparable to [MLA*12,GHFK14]). They supported passers-by to create a wide range of data-driven insights, yet only based on a few of the offered analytical elements.

\subsection{Evaluating Public Data Visualization}

This study demonstrated how models from public display (PD) (e.g. spatial configurations [FH12], engagement levels [MLA*12] and foundations for deployment [PTD*20]), and data visualization (DV) (e.g. insight reporting [SND05], analytical patterns [GW09] and $\log$ analysis [BDF15]), can be combined to provide a more encompassing evaluation of a public visualization. We believe that a rich and mixed-methods evaluation approach is required because in-the-wild situations are subject to various contextual [ $\left.\mathrm{MSH}^{*} 17\right]$ and pragmatic factors [BGR20] that make generalization challenging. As such, this study mapped and combined findings from two disciplines. For instance, we demonstrated that abandoned traces of data exploration (DV) can trigger subsequent use through a detached 'honeypot effect' (PD), that concurrent users socially interact with each other (PD) to negotiate a shared interpretive frame (DV), that titles and entry points (DV) can overcome 'display blindness' (PD), and that personally relevant data (DV) can lead to more discovery engagements (PD).

We also belief that our qualitative results derived from observing, recording and interviewing users during and immediately after their engagement complement established DV findings, ranging from the promoting or inhibiting factors of visualization in casual contexts [ST12] to the impact of visualization titles [KLK18] and explanatory tutorials [BDF15]. 


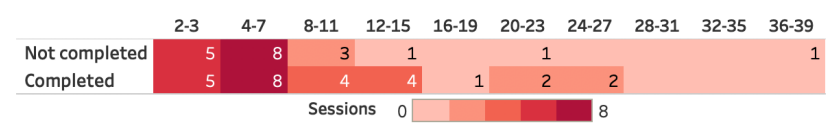

Figure 10: The number of sessions that use more than 3 filters (binned $x$-axis) is higher for those sessions (darker red) in which the explanatory tutorial was completed and not skipped.

\subsection{Meaningful Analytical Interactions}

One's personal familiarity with the data provenance both promoted and inhibited [ST12] passers-by to engage with the public visualization. Due to its location at a sports facility building, the data source (i.e. Strava) sufficiently intrigued some passers-by to try it out. In contrast, people who used competing or no sports tracking tools generally did not feel invited. The crowd-sourced and marketing-influenced nature of the data also provoked users to critically reflect about different types of trust and uncertainty [JS09, MGM19], such as inclusion, cheating or inaccurate sensing, before deciding whether to engage with it.

Takeaway: to maximize the use of a public visualization, its data should be sufficiently familiar to its intended users, and its provenance be neutral, trustworthy and inclusive.

Aside from technical curiosity ("What is that?"), the use of provocative hypotheses as informative titles made some curious to approach the public visualization. The titles were mentioned in $13(n=31)$ audio recordings, typically as the initial point of discussion ("Faster in winter, they ask - Yes, too hot during summer!"). While it is known that informative titles accompanying visualizations can influence [KLK18] and reduce the mental effort and the time it takes to understand [WAJS20] the perceived message of a visualization, we can again [CVM13] confirm how these abilities transfer to a public display setting. Moreover, we show that visualization titles can also help overcome public display blindness by suggesting the value or relevance of the potential insights that can be gained. However, although these hypothetical titles were understood, remembered and sparked social discussions, their influence on the subsequent analytical explorations was low (only 2/31) audio-recorded sessions followed through on the title).

Takeaway: informative titles of public visualizations can inform passers-by of the relevance of its content [SFS12] by its intended analytical purpose, while its imaginative nature can spark interest.

The abandoned screen state of a visualization can encourage passers-by to interact with its display, as video-recorded in 4 instances (and shown in Fig. 6). Following one of the foundations of public display design [PTD*20], our interface was deliberately designed to allow certain unforeseen appropriations. However, we were unaware how the traces of past interaction activities caused a 'detached honeypot effect', as some passers-by possibly felt safer to explore the visualization without having to go through initial screens due to time restrictions (as also suggested by 89 out of 235 sessions skipping the tutorial) or uncertainties ("What is this? Do you know what you have to do?").

Takeaway: each intermediate state of a public visualization could be considered as a potential point of abandonment that then acts as an initial starting view for new users.

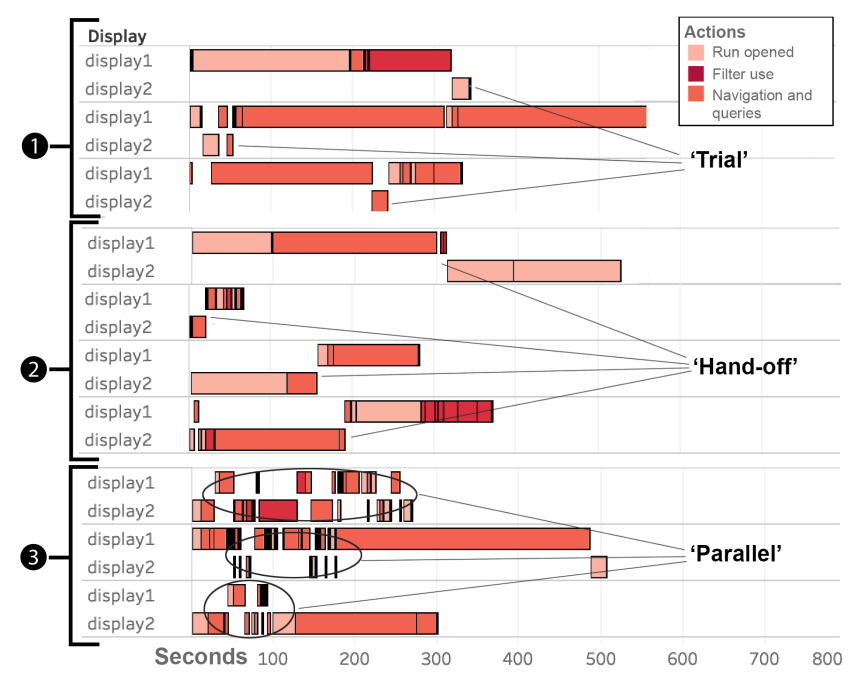

Figure 11: Actions are visualized as blocks of time (seconds) spent in that state. Illustrative examples show use of the two displays in three patterns: (1) 'Trial', (2) 'Hand-offs' and (3) 'Parallel'.

Our results hint that the use of an explanatory tutorial on a public visualization encourages more in-depth analytical sensemaking, as users who followed the tutorial (50/235 sessions, 26 with more than 2 filter uses) felt less overwhelmed by the information density of the visualization, and tended to use its filtering operations more often. Other public visualizations on touch screens and tabletops have similarly found that a narrative structure can facilitate more in-depth engagement [CVM17] but yet still overwhelm casual users [DMMF20]. However, our results stand in contrast to studies that measured the impact of explanatory tutorials in online visualizations, where subsequent user exploration did not significantly increase [BDF15]. Although findings from online media suggest that readers have no clear preference for controlling the progress of a narrative visualization [MHRL*17], public display users seem eager to "skip" this preamble as this option was used in 171/235 sessions.

Takeaway: User preference of explanatory tutorials and their impact on subsequent analytical behavior seems to differ between online and public contexts.

The public visualization was used less for data analytical explorations, as the digital logs contained relatively few instances of the more analytical patterns 'Flip', 'Swap' and 'Drill-Down' (in 15\% of sessions). The frequency of 'Flip' might be reflective of how it required the least cognitive effort to compare and remember its different visual states. In contrast, more in-depth analyses queried specific user runs, often using multiple filters ( 9 sessions depicted in Fig. 8-A,C) to discover detailed insights. While more casual explorations were characterized by shallow inspections of multiple profiles (19 sessions depicted in Fig. 8-B) that focused on more contextual matters like the overall impact of age or the time of day ("The oldest ran less than 6min., well done!"), or unexpected popular data categories like 'season'. Our results thus suggest that the public visualization catered for two distinct user types. Those users with a personal interest in the data actively queried themselves ("I' $m$ 
right there") or their acquaintances ("You know [name]? He looked himself up and he was third!") in order to situate themselves in the community of runners who used an identical trail. Yet other 'information flaneurs ' [DCW11] had a more casual interest in running or sporting in general ("Are there people here who are that fast?"), interpreting the visualization as casual [PSM07] rather than analytical. Although the displays were often used by runners or people familiar with the trail, our evidence thus hints that this analytical behavior would differ if the display was positioned immediately next to its data referent to solely target users of the running trail.

Takeaway: Passers-by can adopt more analytical engagements when the data is personally relevant. Yet passers-by in public space can also find enjoyment in more shallow casual data exploration when the data is relatable.

The predefined profiles were used more frequently than selfinitiated queries ( $80 \%$ of sessions included predefined profiles). This might be because a manual query required more and longer steps, particularly because typing on a public display is challenging ("It's odd to enter your name like this" M, 21) or that the hypothetical labels of the predefined list were more imaginative than finding an acquaintance in the database ("Ha, try comparing with the slowest this week!"). Yet is also suggests how a public visualization may provide alternative interaction strategies that adapt to the time constraints and interests of passers-by [PTD*20] by offering direct entry points that help kick-start the data exploration, similar to what has been explored through crowd-sourcing entry points on online platforms [WK07] or ambient displays [WSP*20]. The most popular entry points focused on human interest (i.e. 'Fastest this week', 101 sessions) rather than demographic profiles (e.g. 'Oldest') Takeaway: Public visualization can overcome potential usability, time and interest constraints by offering predefined and 'frictionless' entry points that attract human interest.

\subsection{Supporting Social Data Engagement}

\subsubsection{Social Interactions Around Public Visualization}

Although we deliberately designed the installation so that collaborative data explorations could be distributed over two separate displays, we found that almost all group negotiations occurred around just one. Concurrent users mainly interacted with each other to jointly align around a shared interpretive frame [ $\left.\mathrm{LKH}^{*} 01\right]$, such as to ascertain how to decode (e.g. the axis "I did well. - No! Oh, [it says] faster and slower") and interact with ("Try it, first name only") the visualization. These conversations were typically sparked by the titles or revolved around the data provenance. Group members transitioned between Trial and Hand-Off patterns to discover the relation between the displays; before shifting from one user sharing or simultaneously interacting on a single display or dispersing over two. In contrast to our pre-design results [CVM20], collaborative analysis was in fact best supported by a single display, a preference that aligns with collaborative work scenarios on shared tabletop displays [IFP* 12$]$.

Takeaway: Concurrent users of a public visualization prefer a single display to jointly build up a shared understanding through discussion before any type of dispersed use might occur.

\subsubsection{Privacy and Ethical Issues}

The required balance of privacy and relevance [PTD*20] on public displays manifested through multiple facets. Selecting a predefined user profile was more popular than querying a custom user profile, such as to compare one's own runs or those of acquaintances. This phenomenon might be easily explained by how only some of passers-by or their acquaintances were included in the data set. As we suggested in [CCVM18], however, publicly visualizing data that directly refers to its intended users also brings about an unexpected social discomfort, because some users feel embarrassed that onlookers might recognize them along with their personal motivation when exploring certain data phenomena ("I could fill in [name] and see data, feels different in the app versus when standing here" $(M, 25)$, "I wonder if it's safe enough..." $(M, 25)$. Others users felt conflicted that the visualization disclosed and even suggested to correlate personal information like weight or gender with physical performance in a public setting ("I understand it's a factor and I'm fine [with it], but maybe not everyone is?" M, 20). One user mentioned the performance data itself as potentially problematic ("Maybe if you're a little slower or you're ashamed of your time, this is not so ideal?" (F, 20).

Takeaway: Critical perspectives of data and its visualization [DFCC13] are more emphasized in public settings. Consider the tension between showing data that is personally relevant to users, and issues of self-representation when the same users are supposed to explore this data in a setting where they can be recognized.

\subsection{Future Public Visualization Opportunities}

The takeaways suggest that there exists an untapped potential for public visualization that is more narrowly focused on specific user expectations, based on its relation to the data referent. In our case, a public visualization that is located immediately next to the running trail could potentially eschew the features that attract the casual information flaneur, such as the hypothetical titles or the predefined user profiles. Vice versa, a public visualization that is more centrally located on the university campus could forgo personalization features in favor of offering more human interest facts.

\section{Conclusion}

We presented the design and in-the-wild evaluation of an analytical and public visualization that was deployed on two linked public displays. Our contributions include a mixed-methods evaluation methodology based on data visualization and public display models, which lead to a collection of actionable takeaways for future design studies. Overall, we think there is great potential in combining the insights from both fields towards making data more accessible in opportunistic settings, particularly where latent user or community interests are reflected in a rich data source that has immediate relevance to a physical location. We thus believe this paper provides new insights and research directions that point to a future in which the opportunistic and situated access to information becomes a socially shared and perhaps even everyday experience.

\section{Acknowledgement}

Funded by the European Union Interreg project Nano4Sports. 


\section{References}

[BClT14] Brehmer M., Carpendale S., Lee B., Tory M.: Predesign empiricism for information visualization: Scenarios, methods, and challenges. In Proceedings of BELIV (2014), ACM, pp. 147-151. doi:10.1145/2669557.2669564.3

[BDF15] Boy J., Detienne F., FEKETE J.-D.: Storytelling in information visualizations: Does it engage users to explore data? Proceedings of CHI (2015), 1449-1458. doi:10.1145/2702123.2702452. 8, 9

[BGR20] Balestrini M., Gallacher S., Rogers Y.: Moving HCI outdoors: Lessons learned from conducting research in the wild. In $\mathrm{HCI}$ Outdoors: Theory, Design, Methods and Applications, McCrickard D. S., Jones M., Stelter T. L., (Eds.). Springer International Publishing, 2020, pp. 83-98. doi:10.1007/978-3-030-45289-6_4.1,8

[BHAR16] Bin Hannan N., Alqahtani F., Reilly D.: JogChalking: Capturing and visualizing affective experience for recreational runners. In Companion Proceedings of DIS (2016), ACM, pp. 129-132. doi:10.1145/2908805.2909406.2

[BLC*14] Bialkowski A., Lucey P., Carr P., Yue Y., SRidharan S., MATTHEWS I.: Identifying team style in soccer using formations learned from spatiotemporal tracking data. In IEEE International Conference on Data Mining Workshop (2014), pp. 9-14. 2

[BOH11] Bostock M., OgIEvetsky V., Heer J.: D3 data-driven documents. IEEE Transactions on Visualization and Computer Graphics 17, 12 (2011), 2301-2309. doi : 10.1109 /TVCG. 2011.185. 4

[BR03] BRIGNull H., Rogers Y.: Enticing people to interact with large public displays in public spaces. In INTERACT (2003), vol. 3, pp. 17-24. 3, 4

[BVgSB14] Behrens M., Valkanova N., Gen. Schieck A. F., BRUMBY D. P.: Smart citizen sentiment dashboard: A case study into media architectural interfaces. In Proceedings of PerDis (2014), ACM, pp. 19:19-19:24. doi:10.1145/2611009.2611036. 2

[CCVM18] Claes S., Coenen J., Vande Moere A.: Conveying a civic issue through data via spatially distributed public visualization and polling displays. In Proceedings of NordiCHI (2018), ACM, pp. 597608. doi:10.1145/3240167.3240206.1,2,10

[CM84] Cleveland W. S., MCGill R.: Graphical perception: Theory, experimentation, and application to the development of graphical methods. Journal of the American statistical association 79, 387 (1984), 531-554. doi:10.1080/01621459.1984.10478080.3

[CM15] Claes S., Moere A. V.: The role of tangible interaction in exploring information on public visualization displays. In Proceedings of PerDis (2015), ACM, pp. 201-207. doi:10.1145/2757710. 2757733. 2

[CMHD10] Crowell H. P., Milner C. E., Hamill J., Davis I. S.: Reducing impact loading during running with the use of real-time visual feedback. Journal of Orthopaedic \& Sports Physical Therapy 40, 4 (2010), 206-213. doi:10.2519/jospt.2010.3166. 2

[CVM13] Claes S., VAnde Moere A.: Street infographics: Raising awareness of local issues through a situated urban visualization. In Proceedings of PerDis (2013), ACM, pp. 133-138. doi:10.1145/ 2491568.2491597. 1, 2, 9

[CVM17] Claes S., VAnde Moere A.: The impact of a narrative design strategy for information visualization on a public display. In Proceedings of DIS (2017), ACM, pp. 833-838. doi:10.1145/ $3064663.3064684 .1,2,9$

[CVM20] COENEN J., VANDE MoERE A.: An analytical visualization of local running activity on a public display. In Companion Proceedings of DIS (2020), ACM, pp. 13-18. doi:10.1145/3393914.3395875. 3,10

[DCW11] DöRK M., CARPEndale S., Williamson C.: The information flaneur: A fresh look at information seeking. In Proceedings of $\mathrm{CHI}$ (2011), ACM, pp. 1215-1224. doi : 10.1145/1978942.1979124. 2,10
[DFCC13] Dörk M., Feng P., Collins C., Carpendale S.: Critical InfoVis: Exploring the politics of visualization. In Proceedings of $\mathrm{CHI}$ Extended Abstracts (2013), ACM, pp. 2189-2198. doi:10.1145/ 2468356.2468739 .10

[DMMF20] DASU K., MA K.-L., MA J., FraZIER J.: Sea of genes: A reflection on visualising metagenomic data for museums. IEEE Transactions on Visualization and Computer Graphics (2020), 1-1. doi : 10.1109/TVCG.2020.3030412.2,9

[FH12] FischeR P. T., HoRneCKER E.: Urban HCI: Spatial aspects in the design of shared encounters for media facades. In Proceedings of $\mathrm{CHI}$ (2012), ACM, pp. 307-316. doi:10.1145/2207676.2207719.1, $3,5,8$

[FTS17] Fredericks J., Tomitsch M., Stewart L.: Design patterns for integrating digitally augmented pop-ups with community engagement. International Journal of E-Planning Research 6, 3 (2017), 19-41. doi:10.4018/IJEPR.2017070102. 2

[GHFK14] Goncalves J., Hosio S., Ferreira D., Kostakos V.: Game of words: Tagging places through crowdsourcing on public displays. In Proceedings of DIS (2014), ACM, p. 705-714. doi:10. $1145 / 2598510.2598514 .8$

[GW09] GotZ D., WEN Z.: Behavior-driven visualization recommendation. In Proceedings of IUI (2009), ACM, pp. 315-324. doi: $10.1145 / 1502650.1502695 .6,8$

[HA08] HeER J., Agrawala M.: Design considerations for collaborative visual analytics. Information Visualization 7, 1 (2008), 49-62. doi:10.1057/palgrave.ivs.9500167. 3

[HSC11] HinRIChS U., Schmidt H., CARPEndale S.: EMDialog: Bringing information visualization into the museum. IEEE Transactions on Visualization and Computer Graphics 14, 6 (2008-11), 1181-1188. doi:10.1109/TVCG.2008.127.2,3

[HTA*03] Huang D., Tory M., Aseniero B. A., Bartram L., Bateman S., Carpendale S., Tang A., Woodbury R.: Personal visualization and personal visual analytics. IEEE Transactions on Visualization and Computer Graphics 21, 3 (2015-03), 420-433. doi:10.1109/TVCG.2014.2359887.2

[HTM*15] Hespanhol L., Tomitsch M., McArthur I., FrederICKS J., SCHROETER R., FOTH M.: Vote as you go: Blending interfaces for community engagement into the urban space. In Proceedings of $C \& T$ (2015), ACM, pp. 29-37. doi:10.1145/2768545.2768553. 2

[HVW09] HeEr J., Viegas F. B., Wattenberg M.: Voyagers and voyeurs: Supporting asynchronous collaborative visualization. Communications of the ACM 52, 1 (Jan. 2009), 87-97. doi:10.1145/ 1435417.1435439 .3

[IFP*12] Isenberg P., Fisher D., Paul S. A., Morris M. R., INKPEN K., CZERWINSKI M.: Co-located collaborative visual analytics around a tabletop display. IEEE Transactions on Visualization and Computer Graphics 18, 5 (2012), 689-702. do i : 10 . 1109/TVCG . 2011. 287. 3,10

[JS09] Johnson C. R., SANDERson A. R.: A next step: Visualizing errors and uncertainty. IEEE Computer Graphics and Applications 23, 5 (2003-09), 6-10. Place: Washington, DC, USA Publisher: IEEE Computer Society Press. doi:10.1109/MCG.2003.1231171. 7, 9

[JWT*20] JANSSEN M., WALraVEns R., ThiBaut E., SChEerder J., BROMBACHER A., VOS S.: Understanding different types of recreational runners and how they use running-related technology. International journal of environmental research and public health 17, 7 (2020). doi:10.3390/ijerph17072276. 2

[KLK18] Kong H.-K., LiU Z., Karahalios K.: Frames and slants in titles of visualizations on controversial topics. In Proceedings of $\mathrm{CHI}$ (2018), ACM. doi:10.1145/3173574.3174012.8,9

[KYY*10] Kaplan O., Yamamoto G., Yoshitake Y., TAKetomi T., SANDOR C., KATO H.: In-situ visualization of pedaling forces on cycling training videos. In SMC (2016-10), IEEE, pp. 994-999. doi : $10.1109 / \mathrm{SMC} .2016 .7844371 .2$ 
[LBV19] LiU C., Balestrini M., Vilaza G. N.: From social to civic: Public engagement with IoT in places and communities. In Social Internet of Things, Soro A., Brereton M., Roe P., (Eds.). Springer International Publishing, 2019, pp. 185-210. doi: 10.1007 / $978-3-319-94659-7 \_10.2$

[LCBll16] Liu C., Chapuis O., Beaudouin-Lafon M., LecolINET E.: Shared interaction on a wall-sized display in a data manipulation task. In Proceedings of CHI (2016), ACM, pp. 2075-2086. doi: $10.1145 / 2858036.2858039 .3$

[LCI*20] Lee B., Choe E. K., Isenberg P., Marriott K., Stasko J. T.: Reaching broader audiences with data visualization. IEEE Computer Graphics and Applications (2020). IEEE. doi : 10.1109/MCG . 2020.2968244 .2

[LHD17] LANGNER R., HORAK T., DACHSELT R.: VISTILES: Coordinating and combining co-located mobile devices for visual data exploration. IEEE Transactions on Visualization and Computer Graphics 24, 99 (2017),1-1. doi:10.1109/TVCG.2017.2744019. 3

[1KE*11] Line C., KAi K., Esko K., GiUlio J., IVAN A., RiIKKA M., KEL: FizzyVis: designing for playful information browsing on a multitouch public display. In Proceedings of the Conference on Designing Pleasurable Products and Interfaces (2011), ACM. doi:10.1145/ 2347504.2347534 .3

[LKH*01] LeE S., Kim S., Hung Y., LAm H., Kang Y., Yi J. S.: How do people make sense of unfamiliar visualizations?: A grounded model of novice's information visualization sensemaking. IEEE Transactions on Visualization and Computer Graphics 22, 1 (2016-01), 499508. doi:10.1109/TVCG.2015.2467195. 7,10

[Lup16] LuPTON D.: The quantified self. John Wiley \& Sons, 2016. 2

[MGM19] Mccurdy N., Gerdes J., Meyer M.: A framework for externalizing implicit error using visualization. IEEE Transactions on Visualization and Computer Graphics 25, 1 (2019), 925-935. do i : 10 . $1109 /$ TVCG.2018.2864913.7,9

[MHRL*17] MCKenNA S., HENRY Riche N., LEE B., BOY J., MEYER M.: Visual narrative flow: Exploring factors shaping data visualization story reading experiences. Computer Graphics Forum 36, 3 (2017), 377-387. doi:10.1111/cgf.13195.9

[MLA*12] Memarovic N., Langheinrich M., Alt F., Elhart I., Hosio S., RUBEGNI E.: Using public displays to stimulate passive engagement, active engagement, and discovery in public spaces. In Proceedings of MAB (2012), ACM, p. 55-64. doi : 10 . 1145/2421076. $2421086.5,8$

[MMF19] MA J., MA K., FraZIER J.: Decoding a complex visualization in a science museum - an empirical study. IEEE Transactions on Visualization and Computer Graphics (2019), 1-1. doi:10.1109/ TVCG.2019.2934401.2

[MSH*17] MäKelä V., Sharma S., Hakulinen J., Heimonen T., TURUNEN M.: Challenges in public display deployments: A taxonomy of external factors. In Proceedings of CHI (2017), ACM, pp. 3426-3475. doi:10.1145/3025453.3025798.8

[MWB*12] MÜller J., Walter R., Bailly G., Nischt M., Alt F.: Looking glass: A field study on noticing interactivity of a shop window. In Proceedings of CHI (2012), ACM, pp. 297-306. doi:10.1145/ $2207676.2207718 .2,5$

[MWE*09] MÜller J., WilmsManN D., EXEler J., BuZECK M., SCHMIDT A., JAY T., KRÜGER A.: Display blindness: The effect of expectations on attention towards digital signage. In Pervasive Computing (2009), Tokuda H., Beigl M., Friday A., Brush A. J. B., Tobe Y., (Eds.), Springer Berlin Heidelberg, pp. 1-8. doi:10.1007/ $978-3-642-01516-8.3$

[OCT*08] Oliveira G., Comba J., Torchelsen R., Padilha M. SILVA C.: Visualizing running races through the multivariate time-series of multiple runners. In Conference on Graphics, Patterns and Images (2013-08), pp. 99-106. doi:10.1109/S IBGRAP I.2013.23. 2
[Off24] OfFEnHUBER D.: Dustmark and ozone tattoos: Autographic displays of air pollution (pictorial). In IEEE VISAP'19 (2019-10-24). URL: https://visap.net/media/Offenhuber-VISAP19.pdf. 2

[OKK*05] OJala T., Kostakos V., KuKKa H., HeikKinen T., LiNDen T., Jurmu M., Hosio S., KRUger F., Zanni D.: Multipurpose interactive public displays in the wild: Three years later. Computer 45, 5 (2012-05), 42-49. doi:10.1109/MC.2012.115.3

[OT15] OFFENHUBER D., TElhan O.: Indexical visualization-the dataless information display. Ubiquitous Computing, Complexity and Culture (2015), 288. doi:10.4324/9781315781129. 2

[PBV09] PERIN C., BOY J., VERNIER F.: Using gap charts to visualize the temporal evolution of ranks and scores. IEEE Computer Graphics and Applications 36, 5 (2016-09), 38-49. Publisher: IEEE. doi : 10 . $1109 /$ MCG. 2016.100 .2

[PSM07] Pousman Z., Stasko J., Mateas M.: Casual information visualization: Depictions of data in everyday life. IEEE Transactions on Visualization and Computer Graphics 13, 6 (2007), 1145-1152. doi : 10.1109/TVCG.2007.70541.10

[PTD*20] PARKer C., TOMitsch M., DaVies N., VAlkanova N., KAY J.: Foundations for designing public interactive displays that provide value to users. In Proceedings of CHI (2020), ACM, pp. 1-12. doi:10.1145/3313831.3376532. 1, 2, 3, 8, 9, 10

[PVS*18] Perin C., Vuillemot R., Stolper C. D., Stasko J. T., WoOd J., CARPENDALE S.: State of the art of sports data visualization. Computer Graphics Forum 37, 3 (2018), 663-686. doi:10.1111/ cgf.13447.2

[PWB20] Perovich L. J., Wylie S. A., Bongiovanni R.: Chemicals in the creek: designing a situated data physicalization of open government data with the community. IEEE Transactions on Visualization and Computer Graphics (2020), 1-1. doi:10.1109/TVCG.2020 . 3030472.2

[RRB*11] Roberts J. C., Ritsos P. D., BADAM S. K., BRODBECK D., KENNEDY J., ElmQVist N.: Visualization beyond the desktop-the next big thing. IEEE Computer Graphics and Applications 34, 6 (201411), 26-34. doi:10.1109/MCG.2014.82.2

[SBB15] Scheerder J., Breedveld K., Borgers J.: Who is doing a run with the running boom? In Running across Europe: The Rise and Size of One of the Largest Sport Markets, Scheerder J., Breedveld K., Borgers J., (Eds.). Palgrave Macmillan UK, 2015, pp. 1-27. doi : 10 . $1057 / 9781137446374 \_1.2$

[SFA14] Steinberger F., Foth M., Alt F.: Vote with your feet: Local community polling on urban screens. In Proceedings of PerDis (2014), ACM, pp. 44:44-44:49. doi:10.1145/2611009.2611015. 2

[SFS12] Schroeter R., Foth M., Satchell C.: People, content, location: Sweet spotting urban screens for situated engagement. In Proceedings of DIS (2012), ACM, pp. 146-155. doi:10.1145/ $2317956.2317980 .1,3,9$

[SND05] SaraiYa P., NorTh C., DUCA K.: An insight-based methodology for evaluating bioinformatics visualizations. IEEE Transactions on Visualization and Computer Graphics 11, 4 (2005), 443-456. doi : $10.1109 /$ TVCG.2005.53.5,8

[ST12] SpRague D., TORY M.: Exploring how and why people use visualizations in casual contexts: Modeling user goals and regulated motivations. Information Visualization 11, 2 (2012), 106-123. doi: $10.1177 / 1473871611433710.8,9$

[STS*12] Stusak S., Tabard A., Sauka F., Khot R. A., Butz A.: Activity sculptures: Exploring the impact of physical visualizations on running activity. IEEE Transactions on Visualization and Computer Graphics 20, 12 (2014-12), 2201-2210. doi : 10 .1109/TVCG . 2014.2352953 .2

[TLR*15] TaYlor A. S., Lindley S., Regan T., Sweeney D., VlachoKyriakos V., Grainger L., Lingel J.: Data-in-place: Thinking through the relations between data and community. In Proceedings of CHI (2015), ACM, p. 2863-2872. doi : $10.1145 / 2702123$. 2702558. 1, 2 
[TWP*06] ThudT A., WALny J., PERIN C., RAJABIYAZdi F., MaCDonald L., Vardeleon D., GReenberg S., CARPEndale S.: Assessing the readability of stacked graphs. In Proceedings of Graphics Interface Conference (2016-06). doi : 10.20380/GI2016.21. 3

[VJTVM13] Valkanova N., Jorda S., Tomitsch M., Vande MoERE A.: Reveal-it!: The impact of a social visualization projection on public awareness and discourse. In Proceedings of CHI (2013), ACM, pp. 3461-3470. doi:10.1145/2470654.2466476.1,2

[VMH12] Vande Moere A., Hill D.: Designing for the situated and public visualization of urban data. Journal of Urban Technology 19, 2 (2012), 25-46. doi:10.1080/10630732.2012.698065.1,2

[VMTH*11] VAnde Moere A., Tomitsch M., Hoinkis M., TrefZ E., Johansen S., Jones A.: Comparative feedback in the street: Exposing residential energy consumption on house façades. In Proceedings of IFIP TC 13 (2011), INTERACT, Springer-Verlag, pp. 470-488. doi:10.5555/2042053.2042103.1,2

[VSBF08] Vos S., Scheerder J., Boen F., Feys J.: A typology of runners: Implications for marketing strategies. In Proceedings of the European Sport Management Conference (2008), pp. 10-13. 2

[WAJS20] Wanzer D. L., AzZam T., Jones N. D., Skousen D.: The role of titles in enhancing data visualization. Evaluation and Program Planning (2020), 101896. doi:https://doi.org/10.1016/j. evalprogplan.2020.101896. 9

[WJD01] Willett W., JANSEN Y., DRAGiCEVIC P.: Embedded data representations. IEEE Transactions on Visualization and Computer Graphics 23, 1 (2017-01), 461-470. doi:10.1109/TVCG.2016. 2598608. 1, 2

[WK09] WOLF G., KELLY K.: Know thyself: Tracking every facet of life, from sleep to mood to pain, 24/7/365, 2009. URL: https:// www.wired.com/2009/06/lbnp-knowthyself/. 2

[WK07] WATTENBERG M., KRISS J.: Designing for social data analysis. IEEE Transactions on Visualization and Computer Graphics 12, 4 (200607), 549-557. doi:10.1109/TVCG.2006.65.10

[WKBF15] WOŹNIAK P., KNAVING K., BJÖRK S., FJELD M.: RUFUS: Remote supporter feedback for long-distance runners. In Proceedings of MobileHCI (2015), ACM, pp. 115-124. doi:10.1145/2785830. 2785893. 2

[Woo07] WoOD J.: Visualizing personal progress in participatory sports cycling events. IEEE Computer Graphics and Applications 35, 4 (201507), 73-81. doi:10.1109/MCG.2015.71.2

[WSP*20] Walny J., Storteboom S., Pusch R., Hwang S. M., Knudsen S., Carpendale S., Willett W.: Pixelclipper: Supporting public engagement and conversation about visualizations. IEEE Computer Graphics and Applications 40, 2 (2020), 57-70. doi: 10 . $1109 /$ MCG. $2020.2968906 .2,10$ 